\title{
Outcomes of social support programs in brain cancer survivors in an Australian community cohort: a prospective study
}

\author{
Khan $\mathrm{F}^{1,2,3, *}$, Amatya $\mathrm{B}^{1}$, Rajapaksa $\mathrm{I}^{1}$ and $\mathrm{Ng} \mathrm{L}^{1,3}$ \\ ${ }^{1}$ Department of Rehabilitation Medicine, Royal Melbourne Hospital, 34-54 Poplar Road, Parkville, Victoria, Australia. \\ ${ }^{2}$ School of Public Health and Preventive Medicine, Monash University, Clayton, Victoria, Australia. \\ ${ }^{3}$ Department of Medicine, Dentistry \& Health Sciences, University of Melbourne, Parkville, Victoria, Australia.
}

\begin{abstract}
This study evaluated the impact of social support programs on improving cancer related disability, neuro-cognitive dysfunction and enhancing participation (quality of life (QoL), social reintegration) in brain tumour (BT) survivors. Participants (n=43) were recruited prospectively following definitive treatment in the community. Each BT survivor received an individualised social support program which comprised: face-to-face interview for education/counselling plus peer support program or community education/counselling sessions. The assessments were at baseline (T1), 6 week (T2) and 6-month (T3) post-intervention using validated questionnaires: depression anxiety stress scale (DASS), functional independence measure (FIM), perceived impact problem profile (PIPP), cancer rehabilitation evaluation system-short form (CARES-SF), a cancer survivor unmet needs measure (CaSUN), McGill quality of life questionnaire (MQOL) and Brief COPE. Participants' mean age was 53 years (range 31-72 years), the majority were female (72\%); median time since BT diagnosis was 2.3 years and almost half (47\%) had high grade tumours. At T2, participants reported higher emotional well-being (DASS 'anxiety' and 'stress' subscales, $\mathrm{p}<0.05$; FIM 'cognition' subscale, $\mathrm{p}<0.01$ ), improved function (FIM 'motor' subscale, $\mathrm{p}<0.01$ ) and higher QoL (CARES-SF 'global' score, $\mathrm{p}<0.05$; MQOL 'physical symptom' subscale, $\mathrm{p}<0.05$ ). At the T3 follow-up, most of these effects were maintained. The intervention effect for BT specific coping strategies emerged for the Brief COPE 'selfdistraction' and 'behavioural disengagement' domains, $(\mathrm{p}<0.05$ for both). There were no adverse effects reported. A post-treatment social support program can improve physical and cognitive function and enhancing overall QoL of BT survivors. Social support programs need further evaluation and should be encouraged by clinicians within cancer rehabilitative services.
\end{abstract}

Keywords: brain neoplasm; social support program; function; cognition; quality of life

\section{Introduction}

Primary brain tumours (BT) affect around 7 in 10,000 people per year worldwide and overall incidence of BT is increasing [1]. Significant medical achievements in the treatment of BT have resulted in a marked increase in the number of survivors and more patients are discharged back to the community [2,3]. Despite medical advances and improved mortality in these persons, a significant percentage of BT survivors experience various combinations of deficits, such as physical, cognitive, psychosocial, behavioural and environmental issues, limiting their daily activity and participation in society $[3,4]$. The treatment regimens itself can produce various adverse effects and can have distressing physical and/ or psychological impact [4]. Patient discharged to the community are confronted by various adjustment issues, such as the patient's perceptions of self-worth, self-image and role reversal within the family, inability to drive and employment issues, financial constraints, marital stress and general limitation on patients' participation [5]. These can have a cumulative effect over time and cause considerable distress to the cancer survivor, their families and reduce quality of life (QoL) (6). Within the

*Corresponding author: Khan F, Department of Rehabilitation Medicine, Royal Melbourne Hospital, 34-54 Poplar Road, Parkville, Victoria, Australia, Tel.: +613 8387 2146; Fax: +613 8387 2222; E-mail: fary.khan@mh.org.au

Received 13 November 2012 Revised 28 January 2013 Accepted 5 February 2013 Published 12 February 2013

Citation: Khan F, Amatya B, Rajapaksa I, Ng L (2013) Outcomes of social support programs in brain cancer survivors in an Australian community cohort: a prospective study. J Cancer Res Ther 1: 24-33. doi:10.14312/2052-4994.2013-4

Copyright: (c) 2013 Khan F, et al. This is an open-access article distributed under the terms of the Creative Commons Attribution License, which permits unrestricted use, distribution and reproduction in any medium, provided the original author and source are credited. 
International Classification of Functioning, Disability and Health (ICF) framework [7], which defines a common language for describing the impact of disease at different levels, BT relatedimpairments (headaches, seizures, neurocognitive dysfunction, muscle weakness, aphasia, visual impairments), can limit 'activity' or function (decreased mobility, inability to self-care) and 'participation' (work, family, social reintegration) and reduce life span [8].

Given the complex multifactorial nature of BT and multiple disabilities encountered by BT survivors, the needs of these persons are best met with a coordinated multidisciplinary and multifaceted approach [5]. This includes acute medical and surgical care, rehabilitation, palliative and other supportive interventions, such as peer or social support. Social support has long been recognised as an important contributor to health and well-being and benefit of social support programs is well established for various conditions, including cancer [9-11]. Social support programs, as a resource have been proposed as an effective means for coping with stressful life experiences and for gaining information and support from others who share a common factor, such as a chronic illness $[12,13]$. Mutual identification, shared experiences and sense of belonging that develops through supportive interventions are thought to impact on psychological outcomes positively [14]. The principal focus of social support programs is to reduce symptoms and limitations on the level of activity and participation (e.g. pain management), psychological distress and to modify 'personal factors' which impact self-management (e.g. self-efficacy and coping style). These impact changes in health status and influence health care utilisation [15].

A systematic review of peer support programs for people with cancer [9] ( $\mathrm{n}=43$ articles) incorporated 5 models of peer support (one-on-one, face-to-face, one-on-one telephone, group face-to-face, group telephone and group Internet) and showed that there was a high level of satisfaction with peer support programs, but the evidence of psychosocial benefit was mixed. Another review [11] of 17 volunteers-delivered peer support programs showed benefits of the program with positive effects for psycho-educational or psychosocial interventions. These interventions resulted in lower rates of anxiety, depression, nausea and pain. Participants had a greater increase in knowledge regarding their disease and treatments, compared to their counterparts not receiving such interventions $[16,17]$.

Despite the plethora of social support programs, there are no published data in BT survivors. The existing data in favour of social support programs for oncological conditions include mainly the breast cancer population [18-21]. Therefore, the objective of this study is to evaluate the impact of social support programs on improving cancer related disability (pain, paralysis), neuro-cognitive dysfunction (anxiety, depression, stress) and enhance participation (QoL, social reintegration, family life, work) in BT survivors; and identify gaps in service provision caused by mismatch between services required and those received by these persons.

\section{Materials and methods}

\section{Setting and participants}

This prospective longitudinal pre-post design study was part of the rehabilitation outcomes research program for BT survivors at the Royal Melbourne Hospital (RMH), a tertiary referral centre in Victoria, Australia and was approved by the hospital ethics committee (HREC no. 2010.216). The RMH program provides rehabilitative and supportive care for inpatient and ambulatory settings, including 6-8 week support programs in the community to promote adaptive and self-management strategies.

The participant selection criteria and methodology have been described previously [22, 23]. Consecutive participants with primary BT (first admission episode only) treated at the RMH between 2007-2011, were recruited from the RMH BT program register. The source of these patients was a pool of persons residing in the community, referred to the RMH from public and private medical clinics across greater Melbourne in Victoria. All participants were aged $>18$ years and fulfilled standard diagnostic criteria for the BT grading system as outlined by the World Health Organization for central nervous system tumours [24]; and assessed by a rehabilitation physician/neurosurgeon at RMH for neurological deficits and clinical assessment for potential benefits of the program. These participants had completed definitive treatment (and were not currently on treatment), resided in the community (area of greater Melbourne $<60 \mathrm{~km}$ radius) and were able to communicate in English. Those who had recurrent or metastatic tumours, significant comorbidities or medically unstable or psychiatric disorders limiting participation in rehabilitation and those bedbound and/or institutionalized in nursing homes were excluded. (Figure 1)

\section{Data collection}

All eligible patients $(n=106)$ were contacted by mail and invited to participate in the project by an independent project officer. Those who replied affirmatively $(n=43)$ were contacted by telephone to explain the study further and recruited [Figure 1]. Once signed consent was obtained, the participants were informed that it could take up to 3 months before they received a support program, consistent with the usual practice. Such a wait time is necessary due to operational issues within a publicly funded hospital (and limited resources) involved in providing the program for all patients at the same time.

This study used a repeat measure design and each subject was prospectively assessed (face-to-face interviews) at baseline, 6 weeks and 6 months following completion of the support program. All interviews and assessments 


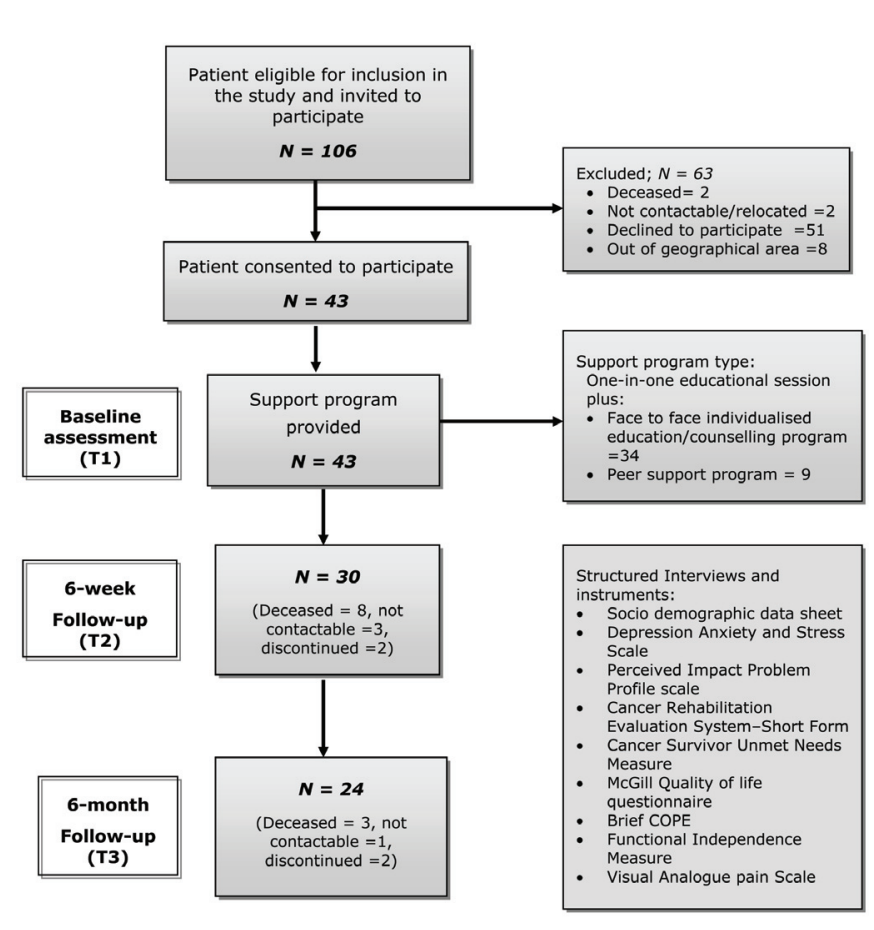

Figure 1 Flow chart of recruitment process

(45 minutes each) were conducted by two independent trained physicians and one research assistant (in clinic or patients' homes), who received 3 half-day training sessions in cognitive and functional ability assessments, using a structured format. The assessors were not in contact with any of the treating team and did not share information about participants or assessments and received separate and different clinical record forms at each interview. Data collected include: demographic and medical information, cognitive and functional ability assessment and health related QoL measures using standardized instruments (see measures). The assessors did not prompt patients, but provided assistance for those who have difficulty with completing the questionnaires. Appropriate rest breaks were provided during these interviews. All assessments were secured and filed and opened at the time of entry into the database by independent data entry officers.

\section{Intervention}

All eligible participants received a one-on-one individualized educational program provided by a rehabilitation physician. In addition, these persons received a tailored social support program comprising either an 8 week group face-to-face peer support program (facilitated by a trained peer support worker) or an educational/counseling intervention program provided by one or more expert (social worker, psychiatrists, psychologists, occupational therapist). These supportive programs were organised and managed through the community therapy service at RMH and designed to augment the traditional rehabilitation approach by targeting the social and emotional consequences of BT. These programs aimed to facilitate psychosocial adjustment and empower participants to openly express their feelings about and seek solutions to their issues and challenges resulting from their conditions. Each program involved a weekly session for a period of 6-8 weeks. All sessions of the peer support program were held at the $\mathrm{RMH}$, while other support programs were held in the community settings for participant convenience. All social support programs were unique and based on participant generated discussions. Prior to the first session, each participant received a brochure by mail, describing the program and providing information on frequently asked questions. The topics were discussed in each program by the rehabilitation physician, psychiatrists, occupational therapists or social worker and included: disease process, personality and behavioural changes, behavioural disturbance management strategies; and coping strategies for functional and psychological disability.

The peer support program was coordinated by a program facilitator, who provided supervision to other peer facilitators where required, during each session to ensure that facilitation standards were met [14]. Apriori compliance for session attendance was set at $60 \%$ and documented by the facilitator. Participants who attended four or more sessions were classed as "completers". Adverse effects of the program were recorded. A dedicated phone number was made available to all study participants five days a week from 9 am to $5 \mathrm{pm}$ to address questions or concerns from patients (and caregivers) and the relevant specialist were contacted as per specific requirements $(\mathrm{FK} / \mathrm{BA})$.

\section{Measures}

At the time of recruitment, baseline assessments were completed (in the patients' homes or in hospital) within a 6 week period. The ICF [7] was used as a conceptual basis for choice of better outcomes for measurement, which provides a framework that describes the impact of disease at the level of impairment, limitation in activity and participation; incorporating contextual (environment and personal) factors.

\section{Participant characteristics and clinical information}

These included socio-demographic and BT-related information (type, localization and stage, treatment status including adverse effects, co-morbid conditions).

\section{Primary Measures}

\section{Measures of societal participation and QoL}

Depression, anxiety stress scale (DASS) [25] - a 21-item instrument, consisting of three 7-item self-report scales was used to measure the negative emotional states of depression, anxiety and stress. Participants rated the extent to which they experienced each state over the past week on a 4-point Likert rating scale. The scores for each domain range from $0-42$, with higher scores indicating more dysfunction. It has good internal consistency [25]. 
The perceived impact of problem profile (PIPP) [26] which consists of 23 items (mobility, self-care, relationships, participation and psychological well-being) was used to assess the impact associated with BT. For each item, respondents were asked to rate "how much impact has your current health problems had on (item of function or activity) using a 6-point scale ('no impact' and 'extreme impact'), with higher scores indicating greater impact.

McGill quality of life questionnaire (MQOL) [27] was used to assessed the QoL, which consist two health related domains (physical well-being and physical symptoms) and three non-health related domains (existential wellbeing, psychological symptoms and support) and one additional single QoL item.

Brief COPE [28] - a 28-item scale was used to assess participants' effective and ineffective coping strategies (active coping, planning, positive reframing, acceptance, humour, religion, using emotional support, using instrumental support, self-distraction, denial, venting, substance use, behavioural disengagement and selfblame).

\section{Primary outcome measures}

Cancer Rehabilitation Evaluation System-Short Form (CARES-SF) [29] - a self-administered measure assessed the QoL. The 59 items in this scale generated a single global score indicating QoL with summary scores for the 5 domains: physical (problems with daily activity), psychosocial (communication and relationship), sexual (interest and performance), marital (problems with a significant relationship) and medical interaction (communication with medical team). The participants rated the degree to which a given problem applied during the 4 weeks prior to the survey using a four point Likert scale $(0=$ not at all to $4=$ very much), with higher scores indicating more difficulty or impairment.

\section{Secondary Measures}

Functional independence measure (FIM) [29] consisting 18 item ( 13 item motor scale and 5 item cognition scale) was used to assess function (activity) and need for assistance, assessing level of function in five subscales: self-care, transfers, locomotion, sphincter control and cognition. Each item was rated on a scale of 1 to 7 ( $1=$ total assistance to $5=$ needs supervision, $6=$ modified independence, 7 =independent). A low score reflects the burden of care in each area measured.

A single item visual analogue pain scale [31] was used to assess pain (score range: $0=$ no pain to $10=$ extreme pain).

Cancer survivor unmet needs measure (CaSUN) [32], consisting 35 need items, six positive change items and an open-response item assessed and identified needs of the BT survivors. Participants choose response options for each item as: no need (0), met the need (1), weak unmet need (2), moderate unmet need (3) and strong unmet need (4). The sum of responses is used to calculate total unmet (2-4) and total need (1-4). Further, the need items are categorised into 5 domains: existential survivorship (e.g., cope with changes to my beliefs); comprehensive cancer care (e.g. complaints addressed), information (e.g. up to date information) and QoL (e.g. changes to my QoL) and relationships (e.g. impact on my relationship).

\section{Statistical analysis}

A series of descriptive analyses (n, \%) were conducted on patient demographics and disease characteristics data. The primary outcome for this study was defined as the impact of the social support program on depression, anxiety and stress (assessed by DASS) [25]. There was no information regarding the 'minimal clinically important difference (MCID)' for DASS in current literature and a change of 8 in the DASS scores in participants was considered clinically relevant. Forty two patients were needed to be recruited for an $80 \%$ chance to detect an 8-point difference in DASS from baseline to 6 months, estimate based on two-tailed $\alpha=0.05$.

Additional analyses were conducted on the subscale scores of the PIPP, MQOL, Brief COPE, CARER-SF, FIM and CaSUN. Given the skewed distribution, primary analyses were conducted using non-parametric tests (Wilcoxon signed rank tests), comparing the pre and post-treatment scores, with the baseline score. Effect size statistics (r) were calculated and assessed against Cohen's criteria (0.1=small, $0.3=$ =moderate, $0.5=$ large effect) [33]. All data was entered twice to avoid errors in data entry and statistical package for social sciences (SPSS), v. 18.0 (SPSS Inc, Chicago, IL) was used for analysis.

\section{Results}

\section{Participant characteristics}

The socio-demographic and disease characteristics of study participants $(n=43)$ are shown in Table 1 . The mean age of the participants was 53 years (range 31-72 years), the majority were female $(72 \%)$ and married (81\%). Median time since BT diagnosis was 2.3 years (Inter Quartile Range, 1.1 to 4.3 years) and almost half (47\%) had high grade BTs (Grade IV) on the WHO tumour grading system. More than half reported pain (56\%), of which $38 \%$ had a headache. Almost one third $(n=13$, $30 \%$ ) reported high levels of depression (measured by DASS). More than two thirds of the sample $(n=30,70 \%)$ reported highest impact on the PIPP 'psychological wellbeing' subscale (scores of $>3$ on the six-point scale) and substantial impact on the PIPP 'participation' subscale $(n=24,56 \%)$, indicating the impact on satisfaction with life, mood, confidence, ability to live independently and ability to participate in work, family, leisure and social activities. Almost one third (31\%) of the participants had caregivers, who either attended or/provided support during the social support programmes (Table 1).

All participants endorsed at least one need (met or unmet, CaSUN). The majority of the participants $(n=37,86 \%)$ 
Table 1 Socio-demographic characteristics of participants $(n=43)$

\begin{tabular}{lc}
\hline \multicolumn{1}{c}{ Characteristics } & $n,(\%)$ \\
Age (years) $\quad$ [Mean (SD), range] & $52.6(11.2), 31-71.9$ \\
Sex Female & $31(72.1)$ \\
Marital status & \\
Married/Partner & $35(81.4)$ \\
Single/Divorced/Separated/Widow & $8(18.6)$ \\
Living with & $7(16.3)$ \\
Alone & $36(83.7)$ \\
Partner/ Family &
\end{tabular}

Education

Secondary
Tertiary

Smokers

Consumes alcohol

$16(37.2)$

Disease duration (years) [Md, (IQR)]

WHO tumour grade* $(n=38)$

Grade I

Grade II

10 (26.3)

Grade III

Grade IV

33 (76.7)

42 (97.7)

Surgery

Type of surgery $(n=42)$

$$
\begin{aligned}
& \text { Debulk } \\
& \text { Complete excision }
\end{aligned}
$$

11 (26.2)

6 (33.3), 1 (5.6)

32 (74.4)

15 (46.9), 4 (12.5)

28 (65.1)

15 (34.9)

24 (55.8)

9 (37.5)

Headaches

Pain score $(0=$ no pain; $10=$ extreme pain $)$

Mean (SD), Range

$2.8(3.1), 0-10$

Pain score $>5$

Limb weakness (MRC motor scale)

$(0=$ no contraction; $5=$ normal power $)$

Left upper limb, Right upper limb, Mean (SD)4.2 (0.8), 4.2 (0.8)

Left lower limb, Right lower limb Mean (SD)4.4 (0.6), 4.4 (0.6)
Characteristics

$n,(\%)$

(unless stated different)

Symptoms

$\begin{array}{lc}\text { Ataxia/incoordination } & 18(41.9) \\ \text { Seizures } & 13(30.2) \\ \text { Paresis } & 10(23.3) \\ \text { Cognitive impairment } & 13(30.2) \\ \text { Visual impairment } & 12(27.9) \\ \text { Dysphasia } & 5(11.6) \\ \text { Dysarthria } & 12(27.9) \\ \text { Sensory-perceptual deficit } & 8(18.6) \\ \text { Bowel/bladder dysfunction } & 5(11.6)\end{array}$

DASS group: $(n, \%)$

Depression

$$
\begin{aligned}
& \text { Normal/mild } \\
& \text { Moderate/severe/extreme severe }
\end{aligned}
$$

13 (30.2)

Anxiety

$$
\text { Normal/mild }
$$

Moderate/severe/extreme severe

5 (11.6) Stress

$$
\text { Normal/mild }
$$

Moderate/severe/extreme severe

$P I P P$ (n, \% recording score of 3 to 6 indicating moderate to extreme impact)

$\begin{array}{lc}\text { Psychological (1-6) } & 30(69.8 \%) \\ \text { Self Care(1-6) } & 8(18.6 \%) \\ \text { Mobility (1-6) } & 13(30.2 \%) \\ \text { Participation (1-6) } & 24(55.8 \%) \\ \text { Relationship (1-6) } & 3(7.0 \%)\end{array}$

CaSUN (Mean (SD), Range)

Total needs

$13.2(6.0), 0-28$

Total unmet needs

6.3 (5.9), 0-26

Total met needs

$6.9(3.4), 0-15$

CaSUN = cancer survivor unmet needs measure; DASS= depression anxiety stress scale, FIM = functional independent measure; $\mathrm{IQR}=$ Interquartile range; $\mathrm{Md}=$ median; $\mathrm{MRC}=$ Medical Research Council; $\mathrm{n}$ = total number; $\mathrm{QoL}=$ quality of life; ROM= Range of Motion; $\mathrm{SD}=$ standard deviation; WHO=World Health Organisation *WHO grading:

Grade I: slow growing, discrete, often surgical cure eg, Astrocytic tumours, meningiomas;

Grade II: slow growing but ability to invade adjacent normal tissue and higher grade of malignancy eg, Oligodendrogliomas;

Grade III: tumours actively reproducing abnormal cells that can infiltrate adjacent cells eg, anaplastic oligodendroglioma; Grade IV: highly malignant and infiltrating into adjacent tissue eg, Glioblastoma 
Table 2 Brain tumour survivors' endorsement of the cancer survivor unmet needs measure (CaSUN) ranked by total need $(\mathrm{n}=43$ )

\begin{tabular}{|c|c|c|c|c|c|c|c|}
\hline \multirow{2}{*}{ Rank } & \multirow{2}{*}{ CaSUN factor } & \multicolumn{2}{|c|}{ Total need } & \multicolumn{2}{|c|}{ Total met need } & \multicolumn{2}{|c|}{ Total unmet need } \\
\hline & & Mean (SD) & Range & Mean (SD) & Range & Mean (SD) & Range \\
\hline 1 & Existential survivorship & $5.1(5.9)$ & $0-28$ & $1.3(1.5)$ & $0-11$ & $3.8(4.5)$ & $0-21$ \\
\hline 2 & Comprehensive cancer care & $3.9(1.5)$ & $0-6$ & $2.8(1.3)$ & $0-5$ & $1.1(1.3)$ & $0-5$ \\
\hline 3 & Information & $2.0(1.2)$ & $0-3$ & $1.6(1.2)$ & $0-3$ & $0.4(0.8)$ & $0-3$ \\
\hline 4 & Quality of life & $0.9(0.7)$ & $0-2$ & $0.4(0.6)$ & $0-2$ & $0.4(0.7)$ & $0-2$ \\
\hline 5 & Relationship & $0.7(0.9)$ & $0-3$ & $0.1(0.3)$ & $0-1$ & $0.6(0.9)$ & $0-3$ \\
\hline
\end{tabular}

CaSUN = Cancer survivor unmet needs measure; $\mathrm{n}=$ total number; $\mathrm{SD}=$ standard deviation

endorsed at least one unmet need, with an average of 6.3 (range 0-26) unmet needs (Table 1). The most endorsed needs were in 'existential survivorship' (mean=5.1, range 0-28) and 'comprehensive cancer care' (mean 3.9, range $0-6$ ). The most endorsed unmet need was also in 'existential survivorship' (mean $=3.8$, range $0-21$ ) and met needs were in 'comprehensive cancer care' (mean $=2.8$, range $0-5$ ) (Table 2).

\section{Social support programs}

All participants completed a one-on-one educational program provided by a rehabilitation physician plus one of the specific social support programs, which included; either a structured peer support program $(n=9,21 \%)$ or an educational/counselling intervention $(\mathrm{n}=34,79 \%)$. The majority of the participants $(n=39,91 \%)$ attended $70 \%$ sessions. During the course of the study 13 patients were lost to follow-up at 6 week assessment ( 8 deceased, 3 not contactable, 2 discontinued as a result of progressive disease) and further 6 were lost at 6-month follow-up (3 deceased, 2 discontinued due to illness, 1 not contactable) (Figure 1) There was no report of adverse effects of social support program at any time.

\section{Short-term subjective outcomes}

At 6 weeks post-treatment follow-up, participants showed statistically significant improvement in psychological functioning (DASS 'anxiety' and 'stress' subscales; $p<0.05$, with small effect size (ES) $r<0.3$ ), CARES-SF 'overall global' score ( $\mathrm{p}<0.05, \mathrm{r}=0.3)$, 'psychological' and 'medical' subscales $(p<0.01, r=0.4$ for both), MQOL 'physical symptom' subscale $(\mathrm{p}<0.05, \mathrm{r}=0.3)$, Brief COPE 'positive framing coping strategy' $(\mathrm{p}<0.01, \mathrm{r}=0.3)$ and in all FIM 'motor' and 'cognition' subscales ( $\mathrm{p}<0.01$ for all), with moderate to large effect sizes ( $\mathrm{r}=0.4$ to 0.5 ). There were no significant, short-term effects in other scores (Table $3)$.

\section{Longer-Term Subjective Outcomes}

At the 6-month follow-up, there were no differences in self-reported cognitive functioning measured by DASS, however, statistically significant increases were still

Table 3 Change scores in subscales for measurement scales overtime

\begin{tabular}{|c|c|c|c|c|c|c|c|}
\hline \multirow[b]{2}{*}{ Scales } & \multirow{2}{*}{$\begin{array}{l}\text { T1 Baseline } \\
M d(I Q R) \\
n=43\end{array}$} & \multirow{2}{*}{$\begin{array}{l}\text { T2 6-week } \\
M d(I Q R) \\
n=30\end{array}$} & \multirow{2}{*}{$\begin{array}{l}\text { T3 6-month } \\
M d(I Q R) \\
n=24\end{array}$} & \multicolumn{2}{|l|}{$Z$ values } & \multicolumn{2}{|c|}{ Effect size } \\
\hline & & & & T1-T2 & T1-T3 & T1-T2 & T1-T3 \\
\hline \multicolumn{8}{|l|}{ DASS } \\
\hline Depression (0-42) & $6(0$ to 16$)$ & $3(0$ to 10$)$ & 4 (2 to 10$)$ & -1.56 & -1.35 & 0.18 & 0.16 \\
\hline Anxiety(0-42) & $2(0$ to 6$)$ & 0 (0 to 2.5$)$ & $0(0$ to 7$)$ & $-2.29 *$ & -0.88 & 0.27 & 0.11 \\
\hline Stress $(0-42)$ & $6(4$ to 14$)$ & $3(0$ to 8.5$)$ & 4 (2 to 9.5$)$ & $-2.25^{*}$ & -0.98 & 0.26 & 0.12 \\
\hline \multicolumn{8}{|l|}{ PIPP } \\
\hline Psychological (1-6) & 3.8 (2.8 to 5$)$ & $3.3(2.8$ to 4.1$)$ & $3.8(2.5$ to 4.6$)$ & -1.22 & -1.17 & 0.14 & 0.14 \\
\hline Self Care(1-6) & 1 (1 to 2.5$)$ & 1 (1 to 1.8$)$ & 1 (1 to 2.2 ) & -0.18 & -1.56 & 0.02 & 0.19 \\
\hline Mobility (1-6) & 2.4 (1.6 to 3.2 ) & 2.1 (1.4 to 3.1$)$ & 2.2 (1 to 3.4 ) & -0.99 & -0.73 & 0.12 & 0.09 \\
\hline Participation (1-6) & 3.4 (2 to.4.4) & 2.6 (2 to 4.3 ) & 3.6 (1.3 to 4.6$)$ & -0.26 & -1.36 & 0.03 & 0.17 \\
\hline Relationship (1-6) & 1.75 (1 to 2.25 ) & 1.4 (1 to 1.8$)$ & 1.1 (1 to 2.9 ) & -1.01 & -0.04 & 0.12 & 0.00 \\
\hline
\end{tabular}




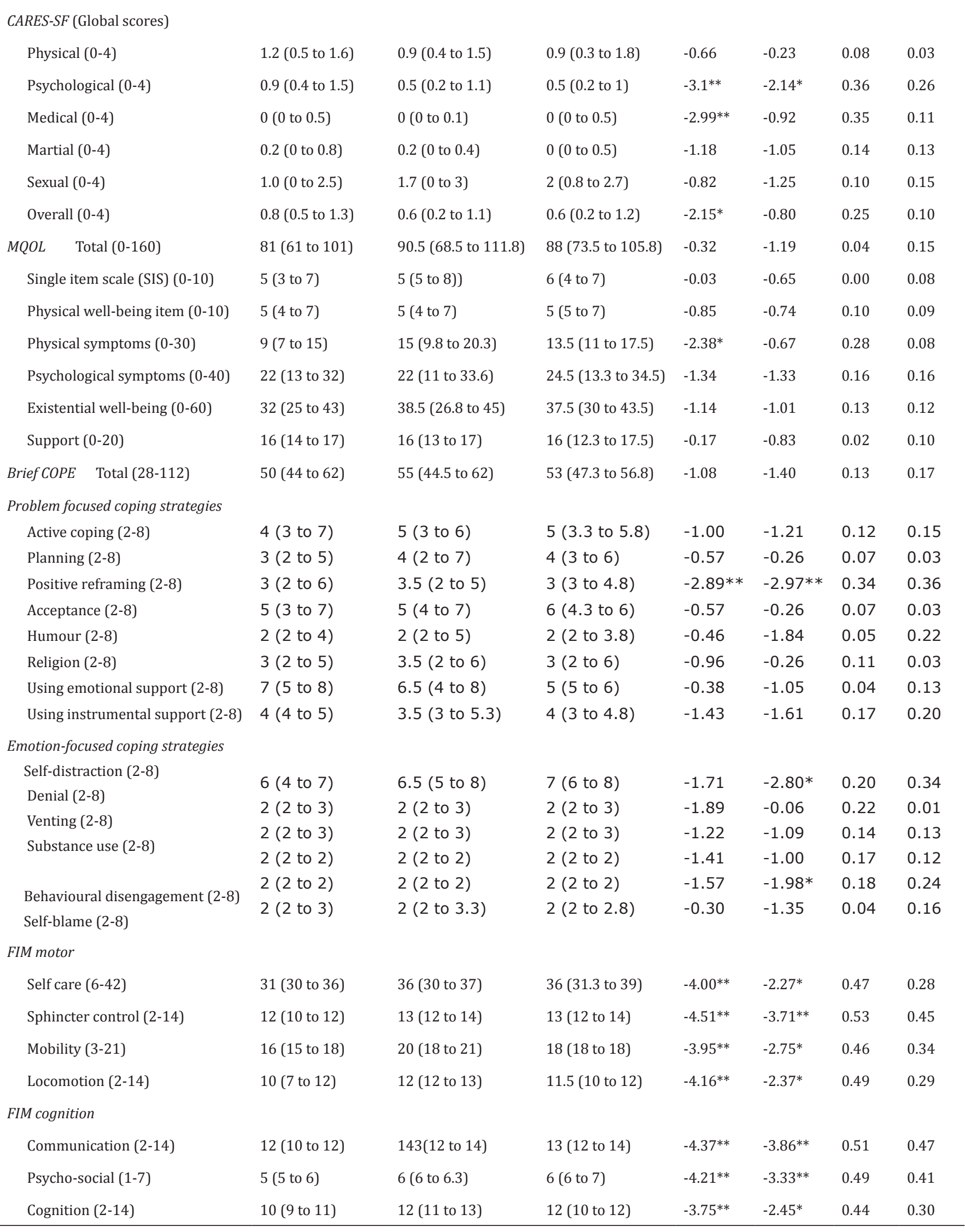

* Correlation significant at the 0.05 level (2-tailed); ${ }^{* *}$ Correlation significant at the 0.01 level (2-tailed)

CARES-SF = cancer rehabilitation evaluation system-short form; CaSUN = cancer survivor unmet needs measure; DASS= depression anxiety stress scale, FIM = functional independent measure; IQR = interquartile range; Md = median; MQOL = McGill quality of life, PIPP = perceived impact of problem profile, $\mathrm{n}=$ total number

\#In scoring MQOL, data were transposed prior to data analysis, where necessary (items 1-3 and 5-8), so that a score of ' 0 ' always indicated the least desirable and ' 10 ' the most desirable situation. For items 1-3, a transposed score of ' 10 ' is assigned when the symptom indicated is "none." 
observed in CARES-SF 'psychological' subscale ( $p<0.05$, $\mathrm{r}=0.3$ ) and Brief COPE 'positive framing coping strategy' $(\mathrm{P}<0.01, \quad \mathrm{r}=0.4)$. Further, a statistically significant improvement in the Brief COPE 'self-distraction' ( $p<0.05$, $r=0.3)$ and 'behavioural disengagement' domains $(\mathrm{p}<0.05, \mathrm{r}=0.2)$ was noted, suggesting improvement in participant's emotion-focused coping strategies. All FIM 'motor' and 'cognition' subscales showed statistically significant improvement ( $p$ between $<0.05$ to $<0.01$ for all, with moderate to large ES, $r=0.3$ to 0.5 ) (Table 3).

\section{Discussion}

To our knowledge this is the first report of the effectiveness of support programs in BT population in an Australian community cohort. The findings from this prospective study suggest that social support programs for BT patients targeting specific behaviour, coping strategies and functional management techniques improved psychological and functional ability and participation. The magnitude of improvement in physical and cognitive function peaked at the 6-week assessment period and was maintained till 6-month review. The participants in this study were similar to those in other studies in terms of age, gender, disease severity and treatment $[3,34,35]$. Supportive care needs are frequently experienced by cancer patients many years after apparently successful treatment [36]. Although most participants in this study seemed to have adjusted well in society, a significant proportion experienced ongoing transient and/or persistent physical and psychosocial morbidity which necessitated the intervention in some form of care need.

The findings of this study could not be compared with others, due to lack of studies in BT population. The positive effects on various aspects of cognitive functions (measured by DASS) at 6-week post-intervention found in this study, was consistent with reports in other cancer subgroups [18, 19, 21, 37-39]. Improvement in functional, cognitive and other outcomes (QoL, coping strategies) was independent of the type of social support program and importantly, achieved irrespective of variability in the duration of these programs. This suggests the need for engagement of these patients in community support activities post-treatment that underpin societal participation and everyday life situation. This provides a platform for developing skills and routines that enhances their daily functional activities, coping strategies and participation, by sharing experiences with persons with or without similar conditions. These findings may have particular importance and relevance for long-term planning and management of this patient population.

A significant proportion of the study participants experienced transient and/or persistent physical and psychosocial morbidities which necessitated some form of intervention for care needs. All participants reported at least one met or unmet need and over three quarters (86\%) reported at least one unmet need. Highest levels of needs endorsed across were in the existential survivorship and comprehensive cancer care domains, consistent with other oncological cohorts such as breast, gynecological, prostate, colorectal [32, 40]. This information has implications for the future planning of clinical service delivery models for improved patient outcomes for cancer survivors [40].

Outcome measurement in cancer research is challenging and varies in different studies [36, 41]. Optimum functional outcome assessment tools for BT survivors are yet to be identified. Generic measures commonly used in practice, may not include all domains relevant for persons with BT and may not be sensitive to change in functional capacity [5]. Instruments such as the FIM do not reflect how the person adapts or copes with the challenges associated with a longer-term disabling condition and has a ceiling effect at a higher level of function [42]. The measurement of QoL is influenced by many factors such physical, psychological and cognitive disabilities and participatory limitations. In addition high mortality rates, often progressive nature and an uncertain prognosis in BT make outcome measurement difficult [22]. Further research is needed to evaluate participatory outcomes in BT population over a longer time.

This study has some potential limitations. First, this is a longitudinal observational study (without a control group), which limits the ability to draw casual relationships between the support program and functional improvements. Second, participants are a selective cohort listed on a database held at a single tertiary institution, with strict inclusion criteria who volunteered to participate, which may limit generalizability of the findings. The study cohort, however, covered a wide geographical population in Victoria and represents a wider sample of BT survivors in the community. Participants in this study were complex in terms of disease severity, symptoms and co-morbidities (reflective of clinical practice). The likelihood of progressive functional decline, the difficulty in psychological adjustment due to constantly changing disability and uncertain prognosis can make a patient's life more challenging [43]. These factors influenced the type and intensity of the intervention provided, this study however, did not attempt to control these factors. The therapists worked individually with each participant to set goals and selected an appropriate tailored social support program. Compliance and attendance in sessions were challenging, but most patients attended $70 \%$ sessions. This study was conducted in real-life settings in busy tertiary public hospital with limited funding. A rigorous study with a control group and single-group based social support program (providing intervention simultaneously to all participants) is planned in the future.

With improved survival rates in BT patients, BT is now accepted as a long-term illness impacting function and participation. Survivorship issues in BT are multifaceted and associated with long-term physical and psychological 
morbidity, however, these morbidities as in other cancer patients can be underestimated [44, 45]. This pilot study showed that community support programs improve functional and cognitive disability in patients with BT with maintenance of benefit for up to 6 months after program completion. Existing literature in BT survivorship recommends multidisciplinary rehabilitation as an integral component of comprehensive care [5], as it improves function and psychological issues [22]. Social support programs as a component of multidisciplinary rehabilitation need further evaluation in well designed clinical trials. Significant variability within the study sample highlights the need for a targeted social support program tailored to the needs and goals of each person over an extended time period.

This exploratory study showed improvements in functional and cognitive outcomes in BT survivors using supportive programs, however these findings need to be further confirmed in randomised and controlled clinical trials. Larger study sample, longer treatment duration and/or follow-ups are required to evaluate the longerterm effects of social support program in this complex patient population.

\section{Ethical approval}

The study was approved by the Royal Melbourne Hospital Ethical Committee and informed consent was obtained from all the subjects.

\section{Financial Disclosure}

F. Khan received the Palliative and Supportive Care Capacity Building Grant provided the Victorian Cancer Agency. No commercial party having a direct financial interest in the results of the research supporting this article has or will confer a benefit upon the authors or upon any organisation with which the authors are associated.

\section{Conflict of interest}

The authors declare that they have no conflict of interest.

\section{Acknowledgement}

This study was conducted in response to the determination of a clinical need by those investigators involved in clinical care. We would like to thank all participants in the study. We thank Ms. L. Oscari for her assistance with interviews and follow-ups. We would also like to thank the peer support program facilitators and community therapists, without whom this study would not have been possible. The above mentioned persons were informed and approve of this acknowledgement.

\section{References}

[1] Flowers A (2000) Brain tumors in the older person. Cancer Control 7:523-538.
[2] Poggi G, Liscio M, Pastore V, Adduci A, Galbiati S, et al. (2009) Psychological intervention in young brain tumor survivors: the efficacy of the cognitive behavioural approach. Disabil Rehabil 31:1066-1073.

[3] Huang ME, Cifu DX, Keyser-Marcus L (2000) Functional outcomes in patients with brain tumor after inpatient rehabilitation: comparison with traumatic brain injury. Am J Phys Med Rehabil 79:327-335.

[4] Tang V, Rathbone M, Park Dorsay J, Jiang S, Harvey D (2008) Rehabilitation in primary and metastatic brain tumours: impact of functional outcomes on survival. J Neurol 255:820-827.

[5] Khan F,Amatya B,NgL,DrummondK, OlverJ(2012)Multidisciplinary rehabilitation for follow-up after primary brain tumour treatment Cochrane Database Syst Rev [in press].

[6] Ness KK, Morris EB, Nolan VG, Howell CR, Gilchrist LS, et al. (2010) Physical performance limitations among adult survivors of childhood brain tumors. Cancer 116:3034-3044.

[7] World Health Organization (2001) International Classification of Functioning, Disability, and Health (ICF). Geneva: WHO.

[8] Ownsworth T, Hawkes A, Steginga S, Walker D, Shum D (2009) A biopsychosocial perspective on adjustment and quality of life following brain tumor: a systematic evaluation of the literature. Disabil Rehabil 31:1038-1055.

[9] Hoey LM, Ieropoli SC, White VM, Jefford M (2008) Systematic review of peer support programs for people with cancer. Patient Educ Couns 70:315-337.

[10] Tilkeridis J, O'Connor L, Pignalosa G, Bramwell M, Jefford M (2005) Peer support for cancer patients. Aust Fam Physician 34:288-289.

[11] Campbell HS, Phaneuf MR, Deane K (2004) Cancer peer support programs-do they work? Patient Educ Couns 55:3-15.

[12] Rootes LE, Aanes DL (1992) A conceptual framework for understanding self-help groups. Hosp Community Psychiatry 43:379-381.

[13] Leavy RL (1983) Social support and psychological disorder: a review. J Community Psychol 11:3-21.

[14] Dennis CL (2003) Peer support within a health care context: a concept analysis. Int J Nurs Stud 40:321-332.

[15] Lorig K, Seleznick M, Lubeck D, et al. (1989) The beneficial outcomes of the arthritis self-management course are not adequately explained by behaviour change. Arthritis Rheum 32: 91-95.

[16] Sheard T, Maguire P (1999) The effect of psychological interventions on anxiety and depression in cancer patients: results of two metaanalyses. Br J Cancer 80:1770-1780.

[17] Devine EC, Westlake S (1995) The effects of psychoeducational care provided to adults with cancer: meta-analysis of 116 studies. Oncol Nurs Forum 22:1369-1381.

[18] Park JH, Bae SH, Jung YS, Kim KS (2012) Quality of life and symptom experience in breast cancer survivors after participating in a psychoeducational support program: a pilot study. Cancer Nurs35: E34-E41.

[19] Power S, Hegarty J (2010) Facilitated peer support in breast cancer: a preand post-program evaluation of women's expectations and experiences of a facilitated peer support program. Cancer Nurs 33:E9-E16.

[20] Cameron LD, Booth RJ, Schlatter M, Ziginskas D, Harman JE, et al. (2005) Cognitive and affective determinants of decisions to attend a group psychosocial support program for women with breast cancer. Psychosom Med 67:584-589.

[21] Rankin N, Williams P, Davis C, Girgis A (2004) The use and acceptability of a one-on-one peer support program for Australian women with early breast cancer. Patient Educ Couns 53:141-146.

[22] Khan F, Amatya B (2012) Factors associated with long-term functional outcomes, psychological sequelae and quality of life in persons after primary brain tumour. J Neurooncolog [in press].

[23] Khan F, Amatya B (2012) Use of the international Classification of Functioning, Disability and Health (ICF) to describe patientreported disability in primary brain tumour in an Australian community cohort J Rehabil Med [in press].

[24] Kleihues P, Burger PC, Scheithauer B (1993) The new WHO classification of brain tumours.Brain Path 3:255-268.

[25] Lovibond SH, Lovibond P. (1995) Manual for the Depression Anxiety Stress Scales. Sydney, Australia: Psychology Foundation of Australia.

[26] Pallant JF, Misajon R, Bennett E, Manderson L (2006) Measuring the impact and distress of health problems from the individual's 
perspective: development of the Perceived Impact of Problem Profile (PIPP). Health Qual Life Outcomes 4: 36.

[27] Cohen SR, Mount BM, Strobel MG, Bui F (1995) The McGill Quality of Life Questionnaire: a measure of quality of life appropriate for people with advanced disease. A preliminary study of validity and acceptability. Palliat Med. 9:207-219.

[28] Carver CS (1997) You want to measure coping but your protocol's too long: consider the Brief COPE. Int J Behav Med 4:92-100.

[29] Ganz PA, Schag CA, Lee TJ, Sims M (1992) The CARES: A generic measure of health related quality of life for patients with cancer. Qual Life Res 1:19-29.

[30] Granger CV (1998) The emerging science of functional assessment: our tool for outcomes analysis. Arch Phys Med Rehabil 79:235240.

[31] Wewers ME, Lowe NK (1990) A critical review of visual analogue scales in the measurement of clinical phenomena. Res Nurs Health 13:227-236.

[32] Hodgkinson K, Butow P, Hunt GE, Pendlebury S, Hobbs KM, et al. (2007) Breast cancer survivors' supportive care needs 2-10 years after diagnosis. Support Care Cancer 15:515-523.

[33] Cohen J (1988) Statistical power analysis for the behavioural sciences.(2nd edn), Hillsdale, NJ: Lawrence Erlbaum Associates.

[34] Bartolo M, Zucchella C, Pace A, Lanzetta G, Vecchione C, et al. (2012) Early rehabilitation after surgery improves functional outcome in inpatients with brain tumours. J Neurooncol 107:537-544.

[35] Huang ME, Wartella JE, Kreutzer JS (2001) Functional outcomes and quality of life in patients with brain tumors: a preliminary report. Arch Phys Med Rehabil 82:1540-1546.

[36] Campbell HS, Phaneuf MR, Deane K (2004) Cancer peer support programs-do they work? Patient Educ Couns 55:3-15.

[37] Gotay CC, Ransom S, Pagano IS (2007) Quality of life in survivors of multiple primary cancers compared with cancer survivor controls. Cancer 110: 2101-2109.

[38] Cameron LD, Booth RJ, Schlatter M, Ziginskas D, Harman JE (2007) Changes in emotion regulation and psychological adjustment following use of a group psychosocial support program for women recently diagnosed with breast cancer. Psychooncology16:171180.

[39] Gotay CC, Moinpour CM, Unger JM, Jiang CS, Coleman D, et al. (2007) Impact of a peer-delivered telephone intervention for women experiencing a breast cancer recurrence. J Clin Oncol 25:20932099.

[40] Hodgkinson K, Butow P, Fuchs A, Hunt GE, Stenlake A, et al. (2007) Long-term survival from gynecologic cancer: psychosocial outcomes, supportive care needs and positive outcomes. Gynecol Oncol104:381-389.

[41] Macvean ML, White VM, Sanson-Fisher R (2008) One-to-one volunteer support programs for people with cancer: a review of the literature. Patient Educ Couns 70:10-24.

[42] Khan F, Amatya B, Pallant JF, Rajapaksa I, Brand C (2012) Multidisciplinary rehabilitation in women following breast cancer treatment: a randomized controlled trial. J Rehabil Med 44:788794.

[43] Kirshblum S, O'Dell MW, Ho C, Barr K (2001) Rehabilitation of persons with central nervous system tumors. Cancer 92:10291038.

[44] Franklin DJ (2007) Cancer rehabilitation: challenges, approaches, and new directions.Phys Med Rehabil Clin N Am 18:899-924.

[45] Huang ME, Sliwa JA (2011) Inpatient rehabilitation of patients with cancer: efficacy and treatment considerations.PMR 3:746-757. 